\title{
Skin Infections in Diabetes: A Review
}

\author{
Gangawane $\mathrm{AK}^{1 *}$, Bhavin Bhatt ${ }^{2}$ and Matkar Sunmeet ${ }^{3}$
}

${ }^{1}$ Dean, Faculty of Applied Sciences, Parul University, Vadodara, Gujarat, India

${ }^{2}$ Faculty of Applied Sciences, Parul University, Vadodara, Gujarat, India

${ }^{3}$ Senior Executive, Arbor Biotech Pvt Ltd, Mumbai, India

\begin{abstract}
Diabetes mellitus (DM) constitutes a growing concern to world population due to the devastating effects of its chronic complications. An estimated number of people with diabetes are projected to rise from 171 million in 2000 to 366 million in 2030. Glucose intolerance is the intermediate stage between normal glucose tolerance and diabetes. It results in ketoacidosis and hyperglycemia causing immune dysfunction. Patients with diabetes mellitus are therefore predisposed to cutaneous infections. If remain unnoticed, undiagnosed in the early stages or not treated properly such infections may get complicated and can even prove lethal. It therefore becomes imperative to recognize signs and symptoms of such infections and approach them appropriately or refer the patient to a diabetologist or dermatologist. Our review literature is a comprehensive communication involving several cutaneous infections that manifest more frequently, with greater severity; or with an elevated risk for complications in patients with diabetes mellitus.
\end{abstract}

Keywords: Diabetes mellitus; Hyperglycemia; Cutaneous infections

\section{Introduction}

Diabetes mellitus (DM), also called Diabetes, comprises a group of metabolic disorders exhibiting high blood sugar levels over an extended period. It is also characterized by state of relative or complete insulin deficiency, leading to gross defects in glucose, fat and protein metabolism. Diabetes is a major endocrine-related disorder causing severe morbidity and mortality all over the world. It constitutes an alarming concern to the global population, largely because of the devastating effects of its chronic complications. In fact, so common and so definite are the chances of developing certain complications during the disease-course that some of them have been considered as 'consequences' rather than complications.

According to WHO report, an estimated number of people with diabetes are projected to rise from 171 million in 2000 to 366 million in 2030. India shall gain ignominious reputation of ranking first (after China and USA) to register 79.4 million diagnosed diabetic cases in 2030 from currently 62 million in 2015. The etiology of diabetes in India is multidimensional including genetic influences combined with environmental factors like lifestyle related obesity and rapid urban migration [1]. Diabetes increases the susceptibility towards plethora of infections. The most common sites of infection in diabetic patients are the skin and urinary tract. Controlled studies carried out by Kass and Hansen establish a strong link (by a factor of two to four) between incidences of bacteriuria in diabetic women than in non-diabetic women $[2,3]$. Other than the infections in urine, upper urinary tract is also involved in approximately $80 \%$ of urinary tract infections in diabetic individuals [4].

Several literatures suggest a strongly positive correlation between hyperglycemia and skin infections.

Patients with DM are more predisposed to skin infections such as folliculitis, furunculosis and subcutaneous abscesses. These infections may appear during the course of the disease or may be the first sign of DM presentation and can also be more severe in these populations [5].

Recent studies on diabetic patients have observed an increased incidence (ranging 20-50\%) of skin infections [6]. Sensory neuropathy, atherosclerotic vascular disease and hyperglycemia all predispose patients with diabetes to skin and soft tissue infections. Such infections can affect any skin surface but most commonly involve the feet [7]. Observations are most common in patients of type-2 diabetes mellitus, often showing poor glycemic control [8]. Foot infections in diabetic patients usually begin in skin ulceration [9].

Bullosis diabeticorum also called (diabetic bulla) is a spontaneous, non-inflammatory, blistering cutaneous condition of acral skin that is unique to patients with diabetes mellitus. It is characterized by a noninflammatory, spontaneous, painless blister, often in acral locations (peripheral body parts, such as feet, toes, hands, fingers, ears or nose) [10].

\section{Physiopathology}

Extensive research has already been undertaken to study the pathogenesis of immune dysfunction in diabetes mellitus. Phagocytic mechanisms like leukocyte chemotaxis and adherence are impaired during hyperglycemia and diabetic acidosis [11].

In antibody-mediated beta cellularis immune system, complement system promotes the opsonization and phagocytosis of non-selfmicroorganisms through macrophages and neutrophils. Complement activation products also induce the second signal for the activation of B-lymphocyte and antibody production. In fact, few studies have recorded a deficiency of the C4 component in DM individuals [12,13].

Not only complement system, even inflammatory cytokine network is found to be having an altered balance. According to some studies, an increased glycation in DM patients is associated with decreased

*Corresponding author: Ajit Gangawane, Dean, Faculty of Applied Sciences, Parul University, Vadodara, Gujarat, India, Tel: 02668-260300/359; E-mail: ajit.gangawane@paruluniversity.ac.in

Received December 23, 2015; Accepted January 29, 2016; Published February 06, 2016

Citation: Gangawane AK, Bhavin Bhatt, Sunmeet M (2016) Skin Infections in Diabetes: A Review. J Diabetes Metab 7: 644. doi:10.4172/2155-6156.1000644

Copyright: $\odot 2016$ Gangawane AK, et al. This is an open-access article distributed under the terms of the Creative Commons Attribution License, which permits unrestricted use, distribution, and reproduction in any medium, provided the original author and source are credited. 
secretion of Interleukin- 1 and Interleukin- 6 by mononuclear cells and monocytes, respectively [14]. Elevated glucose levels have also found to be inhibiting the production of Interleukin- 10 by myeloid cells and Interferon- $\gamma$ and tumor necrosis factor- $\alpha$ by T-cells [15].

The movement of Polymorphonuclear and mononuclear leukocytes, chemotaxis, and phagocytic activity have found to be decreased during hyperglycemia. This hyperglycemic status also interferes in the antimicrobial activity by inhibiting glucose-6phosphate dehydrogenase (G6PD) enzyme thereby elevating apoptosis of polymorphonuclear leukocytes whereas reducing its transmigration through the endothelium. Certain studies performed have shown that when the glycated hemoglobin (HbAlc) is $<8.0 \%$, the proliferative function of $\mathrm{CD} 4^{+} \mathrm{T}-$-lymphocytes and their antigen-response remains impaired [16].

Overall, a compromised and disequilibrium immune system presents the skin of hyperglycemic individual to microbial invasion by both infectious and non-infectious microorganisms.

\section{Skin infections}

Bacterial infections: Patients with DM are at an increased risk of contraction by some bacterial infections. Gram positive bacterial species involving group A and B streptococcal infections by streptococci group, necrotizing fasciitis caused by Group A streptococcus (Streptococcus pyogenes), Staphylococcus aureus, Clostridium perfringens, Bacteroides fragilis, Vibrio vulnificus, Aeromonas hydrophila and acute otitis externa (AOE) by Pseudomonas aeruginosa.

Group A streptococcal infections: It is an infection with group A streptococcus (GAS), that is, Streptococcus pyogenes, the sole bacterial species responsible for a wide range of both invasive and noninvasive infections [17]. In the study carried out by Sharkawy et al. the risk of soft tissue and skin infections contracted with group A streptococcus was found to be almost four times higher in patients with diabetes. In all the study patients soft infections were found to be the most common clinical presentation [18].

Group B streptococcal infections: Other invasive bacterial infections like Group B streptococcus have also been associated with DM. Some studies record Group B streptococcal disease in nonpregnant adults [19]. Skin, soft tissue and bone (cellulitis, foot ulcers and decubitus ulcers) are the frequently infected areas in Group B streptococcus infection. In the study carried out by Schuchat, the risk of Group B streptococcal infections was found to be increased to 11 to 30 -fold in person (age group 20 to 64 years) with diabetes but marginally increased to 3.7-5.7 fold in persons ( $>64$ years) with diabetes, compared with the same age population [20].

Staphylococcal infections: Staphylococcus aureus is a major pathogen implicated in diabetic foot infections [21]. Type-1 diabetic patients show more frequent colonization of the nose and skin by $S$. aureus than non-diabetic and non-insulin-dependent diabetic individuals [22]. Some studies involved chronic stimulation of rabbits with TSST-1 resulting in impaired systemic glucose tolerance. The hallmark finding in this study suggested an active role of $S$. aureus and its superantigens in the progression to type-2 diabetes [23]. Staphylococcal folliculitis or skin abscesses are counted as the most common bacterial infections in uncontrolled diabetes [24].

Acute otitis externa: Invasive ("malignant") otitis externa is a quite rare but potentially fatal infection of the external auditory canal and skull. It mainly involves inflammation of the ear canal $[25,26]$.
Pseudomonas aeruginosa is the causative organism in the vast majority of cases. The acute form is caused primarily by $P$. aeruginosa and $S$. aureus. Acute otitis externa manifests with Unrelenting pain and rapid onset of ear canal inflammation, resulting in otalgia, itching, canal edema, canal erythema, and otorrhea.

It occurs mostly in older patients with diabetes mellitus. It is characterized by purulent discharge, unilateral facial swelling, hearing loss, and granulation tissue in the ear canal. Approximately $86-90 \%$ of patients with otitis externa have concurrent diabetes [27].

Symptoms of acute otitis externa may be mistaken with the symptoms of typical, noninvasive otitis externa. Characteristic clinical feature involves an intense cellulitis and edema of the auditory canal with formation of polypoid granulation tissue. Delay in diagnosis may cause an extension of the infection in cranial osteomyelitis and intracranial involvement [26].

Necrotizing fasciitis: Necrotizing fasciitis (NF) is a rapidly progressive inflammatory infection of the fascia, with secondary necrosis of the subcutaneous tissues. The speed of spread is directly proportional to the thickness of the subcutaneous layer. The perineum, trunk, abdomen and upper extremities are most commonly involved in Necrotizing fasciitis [28].

In soft-tissue infections, foot infections are the most common softtissue infections in patients with diabetes. Among nonpedal soft-tissue infections, necrotizing fasciitis is the most important. The associated mortality in Necrotizing fasciitis is approximately 40 percent. The infection starts in the subcutaneous space and spreads along fascial planes. Necrotizing fasciitis has been classified as type I (infection caused by a combination of anaerobic and one or more facultative aerobic organisms) or type II (caused by group A streptococci, with or without staphylococci) [29-31].

In $\mathrm{DM}$, fasciitis is characterized by poly-microbial growth, with single anaerobic and multiple aerobic microorganisms [32]. Study carried out by Cheng et al. report that the most common (44.5-72.3 $\%$ ) underlying disease in NF patients is Diabetes mellitus (DM) [33].

Fungal and yeast infections: In diabetic individuals, mycotic infections might elevate the risk of manifesting diabetic foot syndrome [34].

Patients with improperly controlled diabetes disease are more prone to Mucocutaneous Candida infections. Infact, Candidal infection (moniliasis) can become a premature indication of undiagnosed diabetes. Perlèche remains atypical warning of diabetes onset in children. Whereas, the correlation between localized candidal infection of the female genitalia (vulvovaginitis) and diabetes have been found strongly correlated [35].

Studies have found out that infections of Candida balanitis, and intertrigo (axillary, inguinal web space) in men are highly indicative of underlying diabetes. Moreover, Glossitis, Paronychia, and onychomycosis are quite frequent [36]. Recent studies have shown a statistically significant relationship between the most common dermatophyte infections like Epidermophyton floccosum and Trichophyton mentagrophytes, Trubrum with diabetes [37].

Few studies suggest that the cases of rhinocerebral mucormycosis (RCM), caused by zygomycetes (Mucor and Rhizipus species) are quite frequently occurring in individuals with diabetes mellitus-mostly with diabetic ketoacidosis [38].

On an average, 50 percent of cases of rhinocerebral mucormycosis 
occur in the diabetic patients. Ketoacidosisis the most critical risk factor in diabetic individuals. The in vitro studies carried out by Gale (1961) documented a lack of inhibitory activity of serum from patients with diabetic ketoacidosis against Rhizopus oryzae that is reestablished upon correction of the acidosis [39].

Viral infections: Since long time, viruses have been suggested as a potential environmental trigger for DM (typically type-1 diabetes). Viruses belonging to the Enterovirus genus have the capacity to initiate and/or accelerate islet autoimmunity, but cannot fully explain the etiology as a sole environmental trigger.

Even though small number of studies carried out, the available data set allows us to interpret with a reasonable degree of confidence, that at least a fraction of DM patients at some point suffered some type of viral insult $[40,41]$.

\section{Conclusion}

Diabetic's skin is more susceptible to skin infections uncommon in non-diabetics. Many of these infections require quick diagnosis and immediate treatment of the severe complications or even fatal outcome is to be averted. With regard to the elevated frequency of skin manifestations in diabetic individual's and considering that they may lead to the development of the obvious disease, specific consideration is advised towards these observations, more precisely in high risk individuals. In addition, superior metabolic control and employing innovative forms of insulin and equipment will help alleviate many of these complications.

\section{References}

1. Kaveeshwar SA, Cornwall J (2014) The current state of diabetes mellitus in India. Australas Med J 7: 45-48.

2. Kass EH (1957) Bacteriuria and the diagnosis of infections of the urinary tract with observations on the use of methionine as a urinary antiseptic. AMA Arch Intern Med 100: 709-714.

3. Hansen RO (1964) Bacteriuria in diabetic and non-diabetic out-patients. Acta Med Scand 176: 721-730.

4. Forland M, Thomas V, Shelokov A (1977) Urinary tract infections in patients with diabetes mellitus. Studies on antibody coating of bacteria. JAMA 238: 1924-1926.

5. Lipsky BA, Tabak YP, Johannes RS, Vo L, Hyde L, et al. (2010) Skin and soft tissue infections in hospitalized patients with diabetes: Culture isolates and risk factors associated with mortality, length of stay and cost. Diabetologia 53 : 914-923.

6. Van Hattem S, Bootsma AH, Thio HB (2008) Skin manifestations of diabetes. Cleve Clin J Med 75: 772, 774, 776-777 passim.

7. Lipsky BA (2004) Medical treatment of diabetic foot infections. Clin Infect Dis 39 Suppl 2: S104-114.

8. Nigam PK, Pande S (2003) Pattern of dermatoses in diabetics. Indian $J$ Dermatol Venereol Leprol 69: 83-85.

9. Lipsky BA (2001) Infectious problems of the foot in diabetic patients. The diabetic foot 6 : 467-480.

10. Aye M, Masson EA (2002) Dermatological care of the diabetic foot. Am J Clin Dermatol 3: 463-474

11. Sahay BK (2013) Infections in Diabetes Mellitus, The Association of Physicians of India Text book of Medicine: Chap 48.

12. Ram S, Lewis LA, Rice PA (2010) Infections of people with complement deficiencies and patients who have undergone splenectomy. Clin Microbiol Rev 23: 740-780.

13. Stoeckle M, Kaech C, Trampuz A, Zimmerli W (2008) The role of diabetes mellitus in patients with bloodstream infections. Swiss Med Wkly 138: 512-519.

14. Geerlings SE, Hoepelman Al (1999) Immune dysfunction in patients with diabetes mellitus (DM). FEMS Immunol Med Microbiol 26: 259-265.
15. Price CL, Hassi HO, English NR, Blakemore Al, Stagg AJ, et al. (2010) Methylglyoxal modulates immune responses: relevance to diabetes. J Cell Mol Med 14: 1806-1815.

16. Peleg AY, Weerarathna T, McCarthy JS, Davis TM (2007) Common infections in diabetes: pathogenesis, management and relationship to glycaemic control. Diabetes Metab Res Rev 23: 3-13.

17. Johansson L, Thulin P, Low DE, Norrby-Teglund A (2010) Getting unde the skin: the immunopathogenesis of Streptococcus pyogenes deep tissue infections. Clin Infect Dis 51: 58-65.

18. Sharkawy A, Low DE, Saginur R, Gregson D, Schwartz B, et al. (2002) Severe group a streptococcal soft-tissue infections in Ontario: 1992-1996. Clin Infect Dis 34: 454-460.

19. Farley MM (2001) Group B streptococcal disease in nonpregnant adults. Clin Infect Dis 33: 556-561.

20. Schuchat A (1998) Epidemiology of group B streptococcal disease in the United States: shifting paradigms. Clin Microbiol Rev 11: 497-513.

21. Rich J, Lee JC (2005) The pathogenesis of Staphylococcus aureus infection in the diabetic NOD mouse. Diabetes 54: 2904-2910.

22. Tuazon CU, Perez A, Kishaba T, Sheagren JN (1975) Staphylococcus aureus among insulin-injecting diabetic patients. An increased carrier rate. JAMA 231 1272 .

23. Bao G, Stach CS, Kulhankova K, Salgado-PabAn W, Klingelhutz AJ, et al. (2015) Chronic Superantigen Exposure Induces Systemic Inflammation, Elevated Bloodstream Endotoxin, and Abnormal Glucose Tolerance in Rabbits: Possible Role in Diabetes 6: e02554.

24. Duff M, Demidova O, Blackburn S, Shubrook J (2015) Cutaneous manifestations of diabetes mellitus. Clin Diabetes 33: 40-48.

25. Zaky DA, Bentley DW, Lowy K, Betts RF, Douglas RG Jr (1976) Malignant external otitis: a severe form of otitis in diabetic patients. Am J Med 61: 298302

26. Slattery WH 3rd, Brackmann DE (1996) Skull base osteomyelitis. Malignant external otitis. Otolaryngol Clin North Am 29: 795-806.

27. Rubin Grandis J, Branstetter BF 4th, Yu VL (2004) The changing face of malignant (necrotising) external otitis: clinical, radiological, and anatomic correlations. Lancet Infect Dis 4: 34-39.

28. Rubin Grandis J, Branstetter BF 4th, Yu VL (2004) The changing face of malignant (necrotising) external otitis: clinical, radiological, and anatomic correlations. Lancet Infect Dis 4: 34-39.

29. Lee YT, Chou TD, Peng MY, Chang FY (2005) Rapidly progressive necrotizing fasciitis caused by Staphylococcus aureus. J Microbiol Immunol Infect 38: 361 364

30. Sentochnik DE (1995) Deep soft-tissue infections in diabetic patients. Infect Dis Clin North Am 9: 53-64.

31. Pessa ME, Howard RJ (1985) Necrotizing fasciitis. Surg Gynecol Obstet 161 357-361.

32. Gozal D, Ziser A, Shupak A, Ariel A, Melamed Y (1986) Necrotizing fasciitis. Arch Surg 121: 233-235.

33. Cheng NC, Tai HC, Chang SC, Chang CH, Lai HS (2015) Necrotizing fasciitis in patients with diabetes mellitus: clinical characteristics and risk factors for mortality. BMC Infect Dis 15: 417

34. Casqueiro J, Casqueiro J, Alves C (2012) Infections in patients with diabetes mellitus: A review of pathogenesis. Indian J Endocrinol Metab 16 Suppl 1: S2736 .

35. Mayser P, Hensel J, Thoma W, Podobinska M, Geiger M, et al. (2004) Prevalence of fungal foot infections in patients with diabetes mellitus type 1 - underestimation of moccasin-type tinea. Exp Clin Endocrinol Diabetes 112 264-268.

36. Saifullah, Mujtaba G (2009) Diabetic's skin: a storehouse of infections. J Pak Assoc Derma 19: 34-37.

37. Lauder N, Binienda J (2005) Case Study: Postsexual Penile Ulcer as a Symptom of Diabetes. Clinical diabetes 4: 191-192.

38. A Hasan M, Fitzgerald SM, Saoudian M, Krishnaswamy G (2004) Dermatology 
Citation: Gangawane AK, Bhavin Bhatt, Sunmeet M (2016) Skin Infections in Diabetes: A Review. J Diabetes Metab 7: 644. doi:10.4172/21556156.1000644

Page 4 of 4

for the practicing allergist: Tinea pedis and its complications. Clin Mol Allergy 2: 5

39. Hsiao YC, Tsan KW, Wang TY (2002) Rhinocerebral mucormycosis in Diabetes: A Case Report. J Intern Med Taiwan 13: 160-164.
40. Gale GR, Welch AM (1961) Studies of opportunistic fungi. I. Inhibition of Rhizopus oryzae by human serum. Am J Med Sci 241: 604-612.

41. Coppieters KT, Boettler T, von Herrath $M$ (2012) Virus infections in type 1 diabetes. Cold Spring Harb Perspect Med 2: a007682. 